\title{
Dynamic Analysis of the Umbilical Cable for Launch \& Recovery System of Seafloor Drill
}

\section{Y.P. JIN}

\author{
College of Mechanical and Electrical Engineering, Central South University, Changsha 410083, China
}

\author{
B.Y. WAN \& D.S. LIU \& Y.D. PENG \\ Hunan Province Engineering Laboratory of Marine Mineral Resources Exploration Equipment and \\ Technology, Hunan University of Science and Technology, Xiangtan 411201, China
}

\begin{abstract}
A launch \& recovery system of a seafloor drill is chosen as the study object, the nonlinear coupling mechanism among the movement of the ship, the umbilical cable, and the seafloor drill caused by irregular wave is investigated, the lumped mass method is used to establish the dynamic model of launch \& recovery system of seafloor drill that considers the influences of the seawater resistance and the elastic deformation of the umbilical cable. Under different sea state conditions, the movement of the seafloor drill and the tension of the umbilical cable are calculated and analyzed. The research results showed that as sea state condition increasing, the amplitude of the movement of the seafloor drill and the tension of the umbilical cable will increase. The variation of the tension of the umbilical cable may lead to the umbilical cable failure and fracture. The analysis results can provide theoretical guidance for the studying of heave compensation and the constant tension automatic control of the launch \& recovery system of seafloor drill.
\end{abstract}

KEYWORD: seafloor drill; umbilical cable; lumped mass method; dynamic analysis

\section{INTRODUCTION}

Seafloor drill is a kind of necessary equipment for the survey of marine mineral resources [1]. In the process of launch \& recovery of the seafloor drill, due to the influence of the wave, ship will produce complex motions, and lead to the seafloor drill motions through the umbilical cable. At the same time, the umbilical cable will cause alternative variation between tension and loosening, which will lead to a significant vibration of the umbilical cable and make the umbilical cable failure and fracture [2$3]$. In order to investigate the internal coupling mechanism, it is necessary to obtain the dynamic response of the seafloor drill movement and the tension of the umbilical cable. Recently, much attention has been paid by many researchers to the mechanism of the movement and the control strategy of the launch \& recovery system [4-5]. A dynamic model of the crane was established based on finite element method, and the calculation results indicate that the large amplitude responses occur when wave period approach the natural period of the payload [67]. However, the payloads have not been in touch with the seawater in the process of launch \& recovery and the elastic deformation of the umbilical cable is often ignored in previous research. In this paper, the seafloor drill needs to launch seafloor to survey the marine mineral resources. In order to analyze the dynamic response of the launch \& recovery system of the seafloor drill and the tension of the umbilical cable, it is necessary to consider the influence of the seawater resistance and the elastic deformation of the umbilical cable.

\section{MODELS FOR LAUNCH \& RECOVERY SYSTEM OF SEAFLOOR DRILL}

\subsection{Model description}

Figure 1 shows a structural model of a launch \& recovery system of seafloor drill, mainly including ship, marine winch, umbilical cable, hoist boom, and seafloor drill.

In Figure 1 three coordinate systems are introduced to describe the launch \& recovery system of the seafloor drill, the inertial reference frame $O_{0}-x_{0} y_{0} z_{0}$ is fixed to the ground. The ship coordinate frame $O-x y z$ is fixed to and, hence, moves with the ship. Point $A$ lies at the rotation axis of the bottom of the hoist boom, and point $B$ lies at the hoisting point of the hoist boom. The distance from point $A$ to origin of the ship coordinate frame is the length of $L_{O A}$, and the distance from point $B$ to point $A$ is the length of $L_{A B}$. $\alpha$ is the angle between $A B$ and the axis $x$.

To describe the orientation of the umbilical cable with respect to the inertial reference frame $O_{0}-x_{0} y_{0} z_{0}$, 
we use a sequence of two angles, represented by $\gamma$ and $\delta$ in Figure 1. These two angles will be referred to as the in-plane and out-of-plane pendulum angles, respectively.

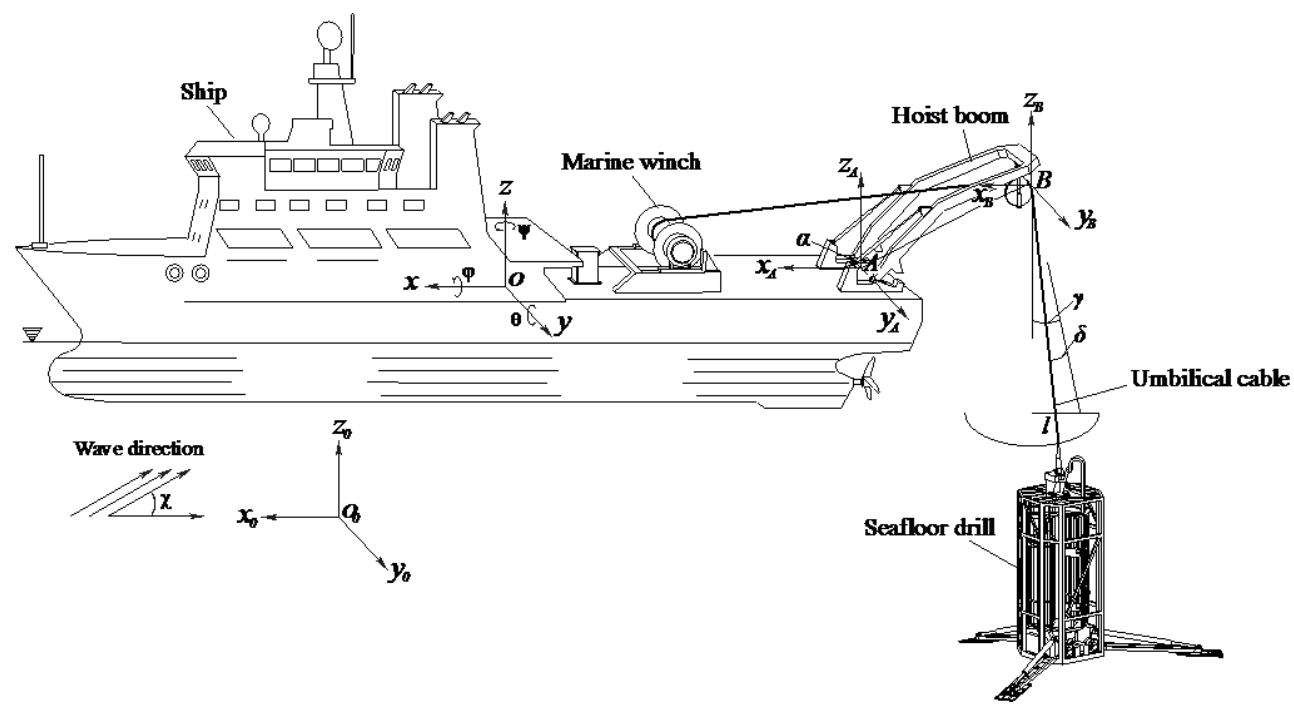

Figure 1. The structural model of launch \& recovery system of seafloor drill

\subsection{Sea state conditions}

The sea elevation at location $x, y$ with respect to the inertial frame $O_{0}-x_{0} y_{0} z_{0}$ is given by[8]

$$
\zeta(x, y, t)=\sum_{i=0}^{N} \zeta_{i} \cos \left[k_{i} x \cos \chi-k_{i} y \sin \chi-\omega_{i} t-\varepsilon_{i}\right]
$$

where $\zeta(x, y, t)$ is the contribution of the wave component $i$ propagating at angle $\chi$ ( $\chi$ is the angle between wave direction and the negative direction of the axis $x$, see Figure 1) with respect to the inertial frame $O_{0}-x_{0} y_{0} z_{0}$ and with a random phase $\varepsilon_{i}$ with uniform distribution on the interval $[0,2 \pi] . \zeta_{i}, \omega_{i}$, and $k_{i}$ are the wave amplitude, wave frequency, and wave number, respectively.

Wave spectrum $S\left(\omega_{i}\right)$ is statistic information of random wave and the relation between $\zeta_{i}$ and $S\left(\omega_{i}\right)$ can be written as

$$
\zeta_{i}=\sqrt{2 S\left(\omega_{i}\right) \Delta \omega_{i}}
$$

Then Eq. (1) can be expressed as

$$
\xi(x, y, t)=\sum_{i=1}^{N} \sqrt{2 S\left(\omega_{i}\right) \Delta \omega_{i}} \cos \left[k_{i} x \cos \chi-k_{i} y \sin \chi-\omega_{i} t-\varepsilon_{i}\right]
$$

Here, A $P-M$ spectrum is selected to describe the wave spectrum. The $P-M$ spectrum is defined as

$$
S(\omega)=\left(0.78 / \omega^{5}\right) \exp \left[-3.12 /\left(\omega^{4} H_{1 / 3}^{2}\right)\right]
$$

where $H_{1 / 3}$ is the significant wave height.

\subsection{Ship motions and external load}

According to the theory of ship sea keeping [8], the differential equations of heaving, rolling, and pitching motions of the ship caused by irregular waves can be described as

$$
\left\{\begin{array}{l}
\left(m_{\text {ship }}+\Delta m_{z z}\right) \ddot{z}+A_{1 z} \dot{z}+A_{2 z} z+A_{3 z} \ddot{\theta}+A_{4 z} \dot{\theta}+A_{5 z} \theta=F_{z} \\
\left(J_{\varphi \varphi}+\Delta J_{\varphi \varphi}\right) \ddot{\varphi}+\mathrm{B}_{1 \varphi}|\dot{\varphi}| \dot{\varphi}+\mathrm{B}_{2 \varphi} \varphi+\mathrm{B}_{3 \varphi} \varphi^{3}=M_{\varphi} \\
\left(J_{\theta \theta}+\Delta J_{\theta \theta}\right) \ddot{\theta}+C_{1 \theta} \dot{\theta}+C_{2 \theta} \theta+C_{3 \theta} \ddot{z}+C_{4 \theta} \dot{z}+C_{5 \theta} z=M_{\theta}
\end{array}\right.
$$

where $A_{1 z}, A_{2 z}, \cdots, A_{5 z} \quad$ are hydrodynamic coefficients of the heaving motion. $B_{1 \varphi}$ is damping moment coefficient of the rolling motion, $B_{2 \varphi}$ and $B_{3 \varphi}$ are restore moment coefficients of the rolling motion. $C_{1 \theta}, C_{2 \theta}, \cdots, C_{5 \theta}$ are hydrodynamic coefficients of the pitching motion. $F_{z}, M_{\varphi}$, and $M_{\theta}$ are the interference forces of the heaving motion, interference moment of the rolling and the pitching motion, respectively. Some coefficients of Eqs.(5) are established according to the experiential formula [9-10].

In the ship coordinate frame $O-x y z$, the hydrodynamic pressure at position $(x, y, z)$ can be expressed as

$$
\Delta p(x, y, z)=-\rho g e^{-k z} \zeta(x, y, t)
$$

Based on Froude-Krylov assumption, the interference force of heaving motion, interference moment of rolling motion and pitching motion are derived as follows

$$
\left\{\begin{array}{l}
F_{z}=\iiint_{V} \frac{\partial \Delta p}{\partial z} d V \\
M_{\varphi}=\iiint_{V}\left(\frac{\partial \Delta p}{\partial y} z-\frac{\partial \Delta p}{\partial z} y\right) d V \\
M_{\theta}=\iiint_{V}\left(\frac{\partial \Delta p}{\partial z} x-\frac{\partial \Delta p}{\partial x} z\right) d V
\end{array}\right.
$$




\subsection{Dynamic equation of launch \& recovery system of seafloor drill}

In this paper, the structural model of the umbilical cable can be viewed as a mass spring damping system (Fig. 2). The point that the umbilical cable contacts with the position of the hoisting point $\mathrm{B}$ of the hoist boom is set to the first node of the umbilical cable.

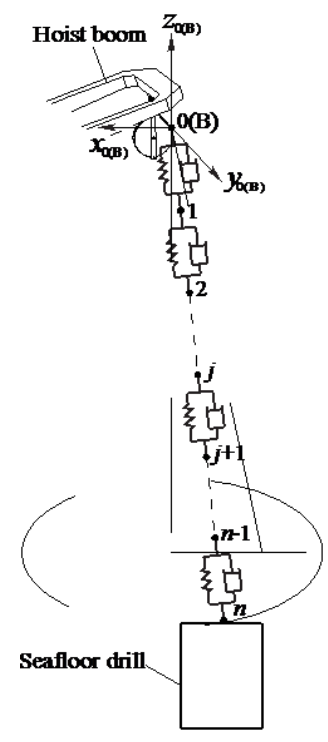

Figure 2. The structural model of umbilical cable

For the node $j\left(x_{j}, y_{j}, z_{j}\right)$ of the umbilical cable, one gets

$$
\left\{\begin{array}{l}
x_{j}=x_{j-1}-l_{j-1, j}^{\prime} \sin \gamma_{j} \cos \delta_{j} \\
y_{j}=y_{j-1}+l_{j-1, j}^{\prime} \sin \delta_{j} \\
z_{j}=z_{j-1}-l_{j-1, j}^{\prime} \cos \gamma_{j} \cos \delta_{j}
\end{array}\right.
$$

where $l_{j-1, j}^{\prime}=\sqrt{\left(x_{j}-x_{j-1}\right)^{2}+\left(y_{j}-y_{j-1}\right)^{2}+\left(z_{j}-z_{j-1}\right)^{2}}, \gamma_{j}$ and $\delta_{j}$ are in-plane and out-of-plane pendulum angles of node $j$ relative to node $j$-1, respectively.

The tension on each node $j$ can be written as

$$
\begin{aligned}
& T_{j}=E A \varepsilon_{j} \\
& \varepsilon_{j}=\left(l_{j-1, j}^{\prime}-l_{j-1, j}\right) / l_{j-1, j}
\end{aligned}
$$

where $l_{j-1, j}$ is the free length of the umbilical cable between node $j-1$ and node $j . E$ and $A$ are the effective Young's modulus and cross-sectional area of the umbilical cable, respectively.

The weight on each node $j$ can be written as

$$
G_{j}=-m_{j} g=-\rho_{\text {cable }} l_{j-1, j} g
$$

where $\rho_{\text {cable }}$ is the linear density of the umbilical cable.

The buoyancy on each node $j$ can be written as

$$
B_{j}=\rho g V_{j}
$$

where $\rho$ is seawater density.
According to Morison Equation, the hydrodynamic drag force on node $j$ can be written as

$$
\left[\begin{array}{c}
D_{j x} \\
D_{j y} \\
D_{j z}
\end{array}\right]=\left[\begin{array}{c}
-\frac{1}{2} \rho C_{n} l_{j-1, j} d\left|\dot{x}_{j}\right| \dot{x}_{j} \\
-\frac{1}{2} \rho C_{n} l_{j-1, j} d\left|\dot{y}_{j}\right| \dot{y}_{j} \\
-\frac{1}{2} \rho C_{t} l_{j-1, j} d\left|\dot{z}_{j}\right| \dot{z}_{j}
\end{array}\right]
$$

where $d$ is the diameter of the umbilical cable. $C_{t}$ and $C_{n}$ are tangential resistance coefficient and normal resistance coefficient of the umbilical cable, respectively.

The dynamic equation of each node of the umbilical cable can be expressed as

$$
\left[\begin{array}{ccc}
m_{j}+m_{a j} & 0 & 0 \\
0 & m_{j}+m_{a j} & 0 \\
0 & 0 & m_{j}
\end{array}\right]\left[\begin{array}{l}
\ddot{x}_{j} \\
\ddot{y}_{j} \\
\ddot{z}_{j}
\end{array}\right]=\left[\begin{array}{c}
T_{j x}+D_{j x} \\
T_{j y}+D_{j y} \\
T_{j z}+G_{j}+B_{j}+D_{j z}
\end{array}\right]
$$

where $j=1,2, \cdots, n-1, \quad m_{a j}$ is the added mass $\left(m_{a j}=k_{a 1} \rho V_{j}\right), \quad k_{a 1}$ is the coefficient of the added mass.

The weight of the seafloor drill can be written as

$$
G_{n}=-m_{\text {drill }} g
$$

The buoyancy of the seafloor drill can be written as

$$
B_{n}=\rho g V_{\text {drill }}
$$

The hydrodynamic drag force on the seafloor drill can be written as

$$
\left[\begin{array}{c}
D_{n x} \\
D_{n y} \\
D_{n z}
\end{array}\right]=\left[\begin{array}{l}
-\frac{1}{2} \rho C_{x} S_{x}\left|\dot{x}_{n}\right| \dot{x}_{n} \\
-\frac{1}{2} \rho C_{y} S_{y}\left|\dot{y}_{n}\right| \dot{y}_{n} \\
-\frac{1}{2} \rho C_{z} S_{z}\left|\dot{z}_{n}\right| \dot{z}_{n}
\end{array}\right]
$$

where $C_{x}, C_{y}$, and $C_{z}$ are the resistance coefficient of movement of the seafloor drill in three directions, $S_{x}, S_{y}$, and $S_{z}$ are resistance surface area of seafloor drill in three directions.

The dynamic equation of the seafloor drill can be expressed as

$\left[\begin{array}{ccc}m_{n}+m_{a n} & 0 & 0 \\ 0 & m_{n}+m_{a n} & 0 \\ 0 & 0 & m_{n}+m_{a n}\end{array}\right]\left[\begin{array}{c}\ddot{x}_{n} \\ \ddot{y}_{n} \\ \ddot{z}_{n}\end{array}\right]=\left[\begin{array}{c}T_{n x}+D_{n x} \\ T_{n y}+D_{n y} \\ T_{n z}+G_{n}+B_{n}+D_{n z}\end{array}\right]$

where $m_{n}$ is the mass of the seafloor drill $\left(m_{n}=m_{\text {drill }}\right), m_{a n}$ is the added mass of the seafloor drill $\left(m_{a n}=k_{a 2} \rho V_{\text {dril }}\right), k_{a 2}$ is the coefficient of the added mass of the seafloor drill. 


\subsection{Initial condition of the umbilical cable}

Since the first node of the umbilical cable was fixed on the hoisting point of the hoist boom, the first node of the umbilical cable shares the same motions with the hoisting point $B$.

Given the ship motions $(z, \varphi, \theta)$, the homogeneous transformation matrix from the ship coordinate frame $O-x y z$ to the inertial coordinate frame $O_{0}-x_{0} y_{0} z_{0}$ is obtained as follows

$\operatorname{Tn}=\left[\begin{array}{cccc}\cos \theta & \sin \theta \sin \varphi & \sin \theta \cos \varphi & x \\ 0 & \cos \varphi & -\sin \varphi & y \\ -\sin \theta & \cos \theta \sin \varphi & \cos \theta \cos \varphi & z \\ 0 & 0 & 0 & 1\end{array}\right]$

Then, the hoisting point $B\left(x_{B}, y_{B}, z_{B}\right)$ of the hoist boom in the inertial coordinate frame $O_{0}-x_{0} y_{0} z_{0}$ is derived as follows

$$
\left[\begin{array}{c}
x_{B} \\
y_{B} \\
z_{B} \\
1
\end{array}\right]=\left[\begin{array}{c}
x-L_{O A} \cos \theta+L_{A B} \cos \alpha \cos \theta+L_{A B} \sin \alpha \sin \theta \cos \varphi \\
y-L_{A B} \sin \alpha \sin \varphi \\
z+L_{O A} \sin \theta-L_{A B} \cos \alpha \sin \theta+L_{A B} \sin \alpha \cos \theta \cos \varphi \\
1
\end{array}\right]
$$

where $z, \varphi$ and $\theta$ are time-varying parameter, $\alpha$ is assumed constant. By differentiating the position of the hoisting point $B\left(x_{B}, y_{B}, z_{B}\right)$, the velocity of hoisting point $B$ can be obtained.

\section{ANALYSIS OF SIMULATION RESULTS}

By applying the former theory, in this section, the calculation and analysis of launch \& recovery system of seafloor drill are carried out based on a scientific research ship. The specifications of sea state are listed in Table 1, and the simulation parameters of launch $\&$ recovery system of seafloor drill are given in Table 2.

Table 1. Sea state conditions

\begin{tabular}{|c|c|c|c|}
\hline Sea state & Level 3 & Level 4 & Level 5 \\
\hline Significant height $H_{1 / 3}(\mathrm{~m})$ & 1.2 & 2.5 & 4.0 \\
\hline Period $T(\mathrm{~s})$ & 4.0 & 6.0 & 8.5 \\
\hline
\end{tabular}

Table 2. Simulation parameters

\begin{tabular}{|l|l|l|l|}
\hline Parameters & Values & Parameters & Values \\
\hline$L(\mathrm{~m})$ & 73.3 & $B(\mathrm{~m})$ & 10.2 \\
\hline$T(\mathrm{~m})$ & 3.4 & $\Delta_{\text {shii }}(\mathrm{kg})$ & 1200000 \\
\hline$m_{\text {dril }}(\mathrm{kg})$ & 8000 & $V_{\text {dril }}\left(\mathrm{m}^{3}\right)$ & 1.56 \\
\hline$L_{O A}(\mathrm{~m})$ & 35.5 & $L_{A B}(\mathrm{~m})$ & 6 \\
\hline$\alpha$ & $135^{\circ}$ & $d(\mathrm{~mm})$ & 32 \\
\hline$E(\mathrm{GPa})$ & 206 & $\rho_{\text {cable }}(\mathrm{kg} / \mathrm{m})$ & 2.35 \\
\hline$C_{x}$ & 1.67 & $C_{v}$ & 1.67 \\
\hline$C_{z}$ & 1.67 & $S_{x}\left(\mathrm{~m}^{2}\right)$ & 8.35 \\
\hline$S_{v}\left(\mathrm{~m}^{2}\right)$ & 8.5 & $S_{z}\left(\mathrm{~m}^{2}\right)$ & 4.67 \\
\hline$C_{n}$ & 1.53 & $C_{t}$ & 0.02 \\
\hline$k_{a 1}$ & 0.5 & $k_{a 2}$ & 0.5 \\
\hline$\rho\left(\mathrm{kg} / \mathrm{m}^{3}\right)$ & 1000 & & \\
\hline
\end{tabular}

In the process of launch \& recovery of the seafloor drill, the ship has been anchored, so we let $v=0$, and let the angle $\chi=30^{\circ}$. For illustration, the seafloor drill at a depth of 1000 meters is introduced as a practical sample.

\subsection{The motion response of the ship}

Figure 3, 4 and 5 respectively show the response curves of the heaving, rolling, and pitching motions of the ship in the sea state 3,4 and 5 conditions. It can be seen that the ship appeared an irregular heaving, rolling and pitching motions under irregular waves. Compared with the significant wave height, the heaving motion of the ship is about one fifth. In addition, by comparing Fig. 4 with 5, the rolling motion is larger than the pitching motion, this is due to the length of the ship is larger than the breadth of the ship, which makes the rolling motion is more stable than the pitching motion. It is also noted that, as sea state condition increasing, the period and the response amplitude of the heaving, rolling and pitching motion of the ship will also be increasing.

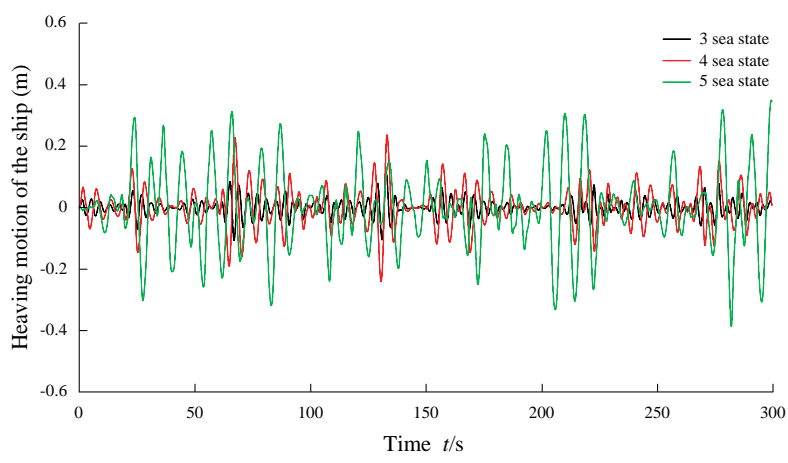

Figure 3. The response curves of the heaving motion

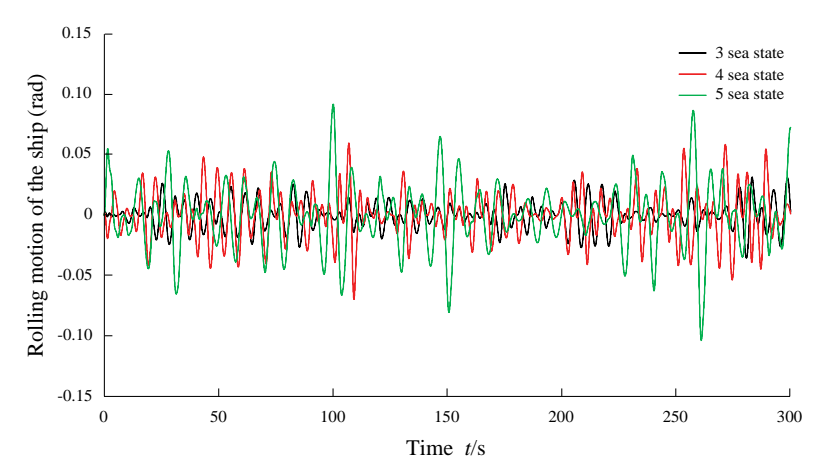

Figure 4. The response curves of the rolling motion

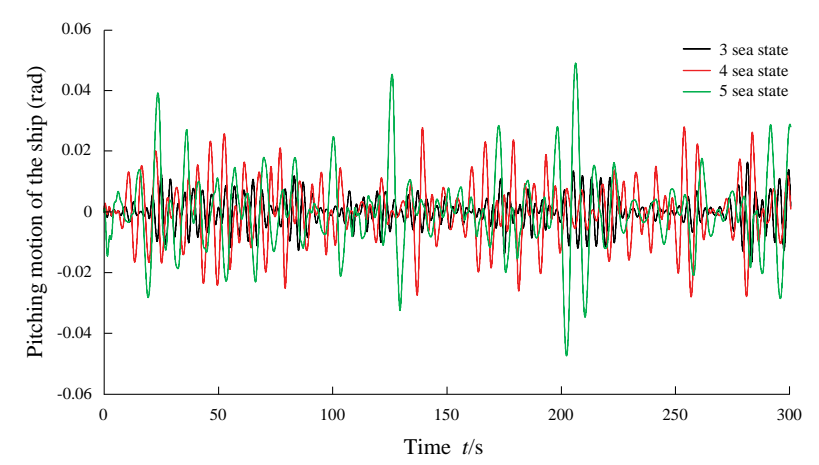

Figure 5. The response curves of the pitching motion 


\subsection{Dynamic analysis of launch \& recovery system of the seafloor drill at a depth of 1000 meters}

Figures 6, 7 and 8 show the response curves of the in-plane, out-of-plane pendulum angle $(\gamma$ and $\delta$ ) of the umbilical cable and the displacement in the $x, y$ direction of the seafloor drill, respectively. Form Figures 6,7 and 8 we can see that the in-plane and out-of-plane pendulum angles $(\gamma$ and $\delta$ ) of the umbilical cable and the displacement in the $x, y$ direction of the seafloor drill are enhanced along with the increase of the sea state condition.

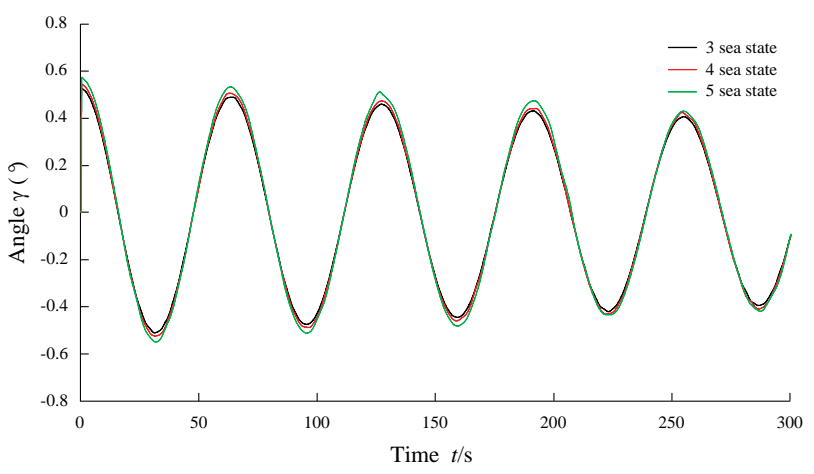

Figure 6. The response curve of pendulum angle $\gamma$

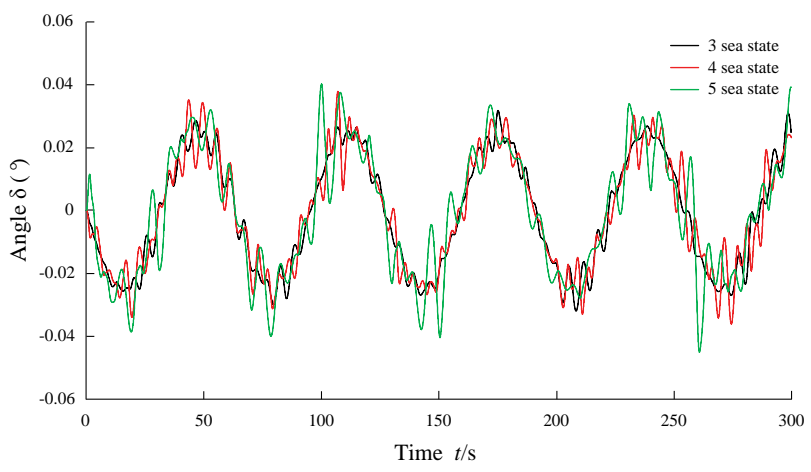

Figure 7. The response curve of pendulum angle $\delta$

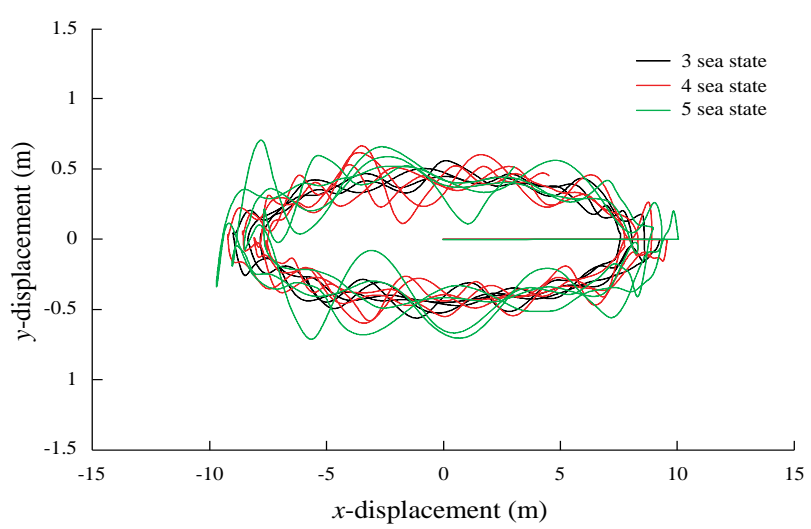

Figure 8 . The $x, y$ displacement of the seafloor drill

Figure 9 shows the response curves of the heaving motion of the seafloor drill. Compared with the maximum amplitude of the heaving motion of the ship, the maximum amplitude of the heaving motion of the seafloor drill is more than times larger than it. This is due to the hoist boom was fixed in the quarter-deck of the ship, which make the pitching motion of the ship has the most significant influence on the heaving motion of the seafloor drill.

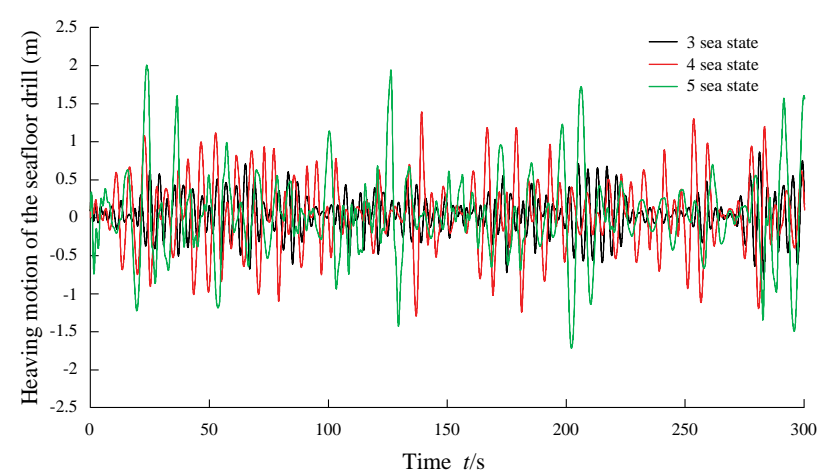

Figure 9. The heaving motion of the seafloor drill

Figure 10 shows the response curves of the tension of the umbilical cable. Form Figure 10 we can see that the tension of the umbilical cable is increased along with the increase of the sea state condition.

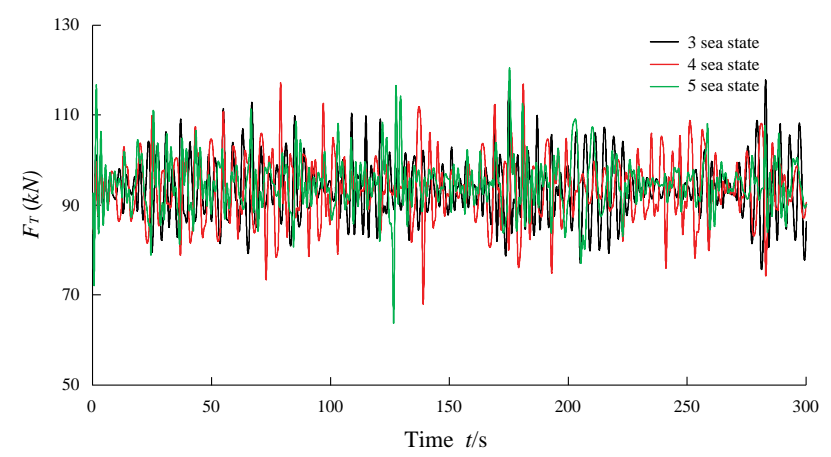

Figure 10. The tension of the umbilical cable

\section{CONCLUSIONS}

A lumped mass method is used to establish the dynamic model of launch \& recovery system of seafloor drill that considers the influences of the seawater resistance and the elastic deformation of the umbilical cable. Under different sea state conditions, the movement of the seafloor drill and the tension of the umbilical cable are calculated and analyzed.

The research results show that as the increasing of the sea state condition, the amplitude of the in-plane and out-of-plane pendulum angles of the umbilical cable, the displacement in the $x$ and $y$ direction of the seafloor, the heaving motion of the seafloor drill and the tension of the umbilical cable will increase. The analysis results can provide theoretical guidance for the studying of heave compensation and the constant tension automatic control of the umbilical cable.

\section{ACKNOWLEDGMENT}

This work is supported by the National High Technology Research and Development Program of 
China ("863" Program) (Grant No. 2012AA091301), Science \& Technology Project of Hunan Province (Grant No. 2014FJ1004), and the Natural Science Combined Foundation with Province and City of Hunan Province (Grant No. 2015JJ5029).

\section{REFERENCES}

[1] Rahul S. 2011. Deep sea mining economic, technical, technological, and environmental considerations for sustainable development. Marine Technology Society Journal. 5(5): 28-41.

[2] Wang P C, Fang Y C, Xiang J L, et al. 2011. Dynamics Analysis and Modeling of Ship-mounted Boom Crane. Journal of Mechanical Engineering. 17 (20): 34-39.

[3] Yoshiki N. 2012. Static analysis of axially moving cables applied for mining nodules on the deep sea floor. Applied Ocean Research.34: 45-51.
[4] Chin C, Nayfeh A H, Abdel-Rahman E. 2001. Nonlinear dynamics of a boom crane. Journal of Vibration and Control. 7(2): 199-220.

[5] Hanspeter S. 2008. Rate-based ship-mounted crane payload pendulation control system. Control Engineering Practice.16:132-145.

[6] Katrin E, Edwin K. 2003. Nonlinear Dynamics in the Motion of Floating Cranes. Multibody System Dynamics. 9: 377-387.

[7] Ran H L, Wang X L, Hu Y J, et al. 2009. Dynamic Response Analysis of Moored Crane-ship with Flexible Booms. Journal of Mechanical Engineering. 45(10): 42-46.

[8] Li J D. Ship seakeeping. Harbin engineering university press, 2007.

[9] Tasai F. 1961. Damping Force and Added Mass of Ships Heaving and Pitching. Transactions of the West Japan Society of Naval Architects. 21: 109-132.

[10]Fossen T I, Smogeli $\varnothing$ N. 2004. Nonlinear Time-Domain Strip Theory Formulation for Low-Speed Manoeuvring and Station-Keeping. Modeling, Identification and Control. 25(4), 201-221. 